\title{
Adaptive Route Choice Models in Stochastic Time-Dependent Networks
}

\author{
S. Gao \\ E. Frejinger \\ M. Ben-Akiva \\ July 7, 2007
}

Report TRANSP-OR 070730

Transport and Mobility Laboratory

School of Architecture, Civil and Environmental Engineering

Ecole Polytechnique Fédérale de Lausanne

transp-or.epfl.ch 


\begin{abstract}
We study adaptive route choice models that explicitly capture travelers' route choice adjustments according to information on realized network conditions in stochastic time-dependent networks. Two types of adaptive route choice models are explored: an adaptive path model where a sequence of path choice models are applied at intermediate decision nodes; and a routing policy choice model where the alternatives correspond to routing policies rather than paths at the origin. A routing policy in this paper is a decision rule that maps from all possible (node, time) pairs to next links out of the node. A policy-size Logit model is proposed for the routing policy choice, where policysize is a generalization of path-size in path choice models to take into account the overlapping of routing policies. The specifications of estimating the two adaptive route choice models are established and the feasibility of estimation from path observations is demonstrated on an illustrative network. Prediction results from three models non-adaptive path model, adaptive path model, and routing policy model - are compared. The routing policy model is shown to better capture the option value of diversion than the adaptive path model. The difference between the two adaptive models and the non-adaptive model is larger in terms of expected travel time, if the network is more stochastic, indicating that the benefit of being adaptive is more significant in a more stochastic network.
\end{abstract}

\title{
1 Introduction
}

Transportation systems are inherently uncertain due to disturbances such as incidents, vehicle breakdowns, work zones, bad weather conditions, special events and so forth. On the other hand, real-time information in various

formats is available, from personal observations, websites, variable message signs (VMS), radio broadcasts, cell phones to personal in-vehicle systems. Real-time information can reduce the uncertainty of the traffic network, and therefore potentially help traveler make better route choice decisions. Travelers usually obtain updated information at various decision points during a trip, and potentially a route choice is made at each of the points 
based on updated perception of the uncertain network. This dynamic process of a series of route choices with the presence of real-time information in a stochastic network, is of great interest, since it is critical to the evaluation of any real-time information system. We refer to models that capture this process as adaptive route choice models, in that the choices are adapted to the realized network conditions revealed by real-time information.

Most discrete choice models for route choice analysis are based on deterministic networks. Examples of such models are Path Size Logit (Ben-Akiva and Ramming, 1998; Ben-Akiva and Bierlaire, 1999), C-Logit (Cascetta et al., 1996), Cross-Nested Logit (Vovsha and Bekhor, 1998), and Logit Mixture (Ramming, 2001; Bekhor et al., 2002; Frejinger and Bierlaire, 2007). In this paper we refer to these models as non-adaptive path choice models because travelers are assumed to make their complete path choice at the origin. The fact that travelers adjust their route choices en-route in response to revealed traffic conditions is therefore ignored.

A seemingly natural way to build adaptive route choice models is to have a sequence of path choice models at decision nodes, where the attributes of alternative paths to the destination reflect updated information. Any of the above mentioned route choice models with adequate incorporation of real-time information could in principle be applied successively in a stochastic network to model adaptive route choice behavior. DynaMIT (Ben-Akiva et al., 2002) and DYNASMART (Mahmassani, 2001) are examples of dynamic traffic assignment models that apply an adaptive path choice model. Calibration of DynaMIT's route choice model based on field data is reported in Balakrishna (2006) and Balakrishna et al. (2007).

There have been a large number of studies of path choice models with real-time information, both pre-trip and en-route, and a recent literature review can be found in Abdel-Aty and Abdalla (2006). Some models predict the decision to switch from a previous chosen or experienced route (e.g. Polydoropoulou et al., 1996; Abdel-Aty and Abdalla, 2004; Mahmassani and Liu, 1999; Srinivasan and Mahmassani, 2003); others are route choice models with explicit paths as alternatives (e.g. Bogers et al., 2005; Peeta and Yu, 2005; Abdel-Aty and Abdalla, 2006).

Information is usually modeled by adding attributes to a model spec- 
ification. The attributes can be binary to indicate whether certain information is available or not (e.g. Abdel-Aty and Abdalla, 2004; Polydoropoulou et al., 1996; Srinivasan and Mahmassani, 2003; Abdel-Aty and Abdalla, 2006), proxies such as queue length (Bogers et al., 2005) or travel time (Mahmassani and Liu, 1999; Srinivasan and Mahmassani, 2003) or fuzzy variables with continous membership functions (Peeta and Yu, 2005). Most of these models were estimated with interactive simulation data or synthetic data (with the exception of Polydoropoulou et al. (1996)), which suggests the difficulty of obtaining field data. Among the models, Srinivasan and Mahmassani (2003) and Abdel-Aty and Abdalla (2006) use observations from all decision nodes during a trip to estimate the models, which could be treated as panel data, while others use cross-sectional data which do not consider the successive route choice adjustment during a trip.

In this paper, we propose a novel adaptive route choice model where the alternatives are not paths, but routing policies. Generally speaking, a routing policy is a decision rule that maps all possible network states in a stochastic network to decisions, while a path is a fixed set of links without incoporating information or stochasticity. The definition of a routing policy depends on the underlying stochastic network and the information access (Gao and Chabini, 2006). Some researchers refer to it as strategy, hyperpath or online path with recourse. The literature includes a numbers of algorithmic studies of optimal routing policy problems (e.g. Hall, 1986; Polychronopoulos and Tsitsiklis, 1996; Marcotte and Nguyen, 1998; Pretolani, 2000; Miller-Hooks and Mahmassani, 2000; Miller-Hooks, 2001; Waller and Ziliaskopoulos, 2002; Gao, 2005; Gao and Chabini, 2006), however econometric models of routing policy choice is a new area. Ukkusuri and Patil (2006) applied sequential logit loading of hyperpath flows in an equilibrium traffic assignment, where travelers were assumed to learn realized travel times on outgoing links. However the estimation problem was not addressed. This paper therefore is the first research effort to develop an estimator of a routing policy choice model and demonstrate the feasibility of estimating such a model.

The paper is organized as follows. Background information on adaptive path choice and routing policy choice is presented in Section 2, while Sec- 
tion 3 gives an illustrative example to clarify the concepts and illustrate the difference between the two adaptive models. In Section 4 we formulate the estimation problems of adaptive path choice and routing policy choice in a stochastic time-dependent network, where the observations are manifested paths, and the choice of routing policy is latent. Section 5 contains the numerical experiment setup, estimation results of three models - nonadaptive path, adaptive path and routing policy - from synthetic data and discussions of prediction results to gain insights into the adaptive route choice models. Conclusions and future research directions are provided in Section [6.

\section{Background}

We study adaptive route choice models in a stochastic time-dependent network, where the travel time on each link $\ell=(v, w)$, with source node $v$ and sink node $w$, for an arrival time $t$ at $v$ is a random variable $\widetilde{T}_{\ell, t}$ with finite number of discrete, positive and integral support points. A support point is defined as a distinctive value (vector of values) a discrete random variable (vector) can take, and thus the probability mass function (PMF) of a random variable (vector) is the combination of support points and the associated probabilities. In this paper, a symbol with a $\sim$ over it is a random variable, while the same symbol without the $\sim$ is one specific value of the random variable, which sometimes might be superscripted with an index for support point. Since link travel time is random, a traveler entering a link at a given time might exit the link at different times, which might result in different travel time PMFs on the next link. A traveler with a chosen path will take a fixed link out of an intermediate node regardless of the possible different arrival times at the node. In another word, a path is purely topological. On the other hand, if a traveler has a priori knowledge of the PMFs of time-dependent link travel times as well as the realized arrival times at nodes, he/she can make adaptive route choices accordingly.

Two types of adaptive route choice models are studied in this paper: adaptive path model and routing policy model. Travelers are assumed to maximize their utility, which can be a combination of expected travel time, 
travel time standard deviation and other attributes. In the adaptive path model, at each intermediate decision node and for each possible arrival time, the traveler selects among a set of paths to the destination, and takes the first link of the chosen path. Once the traveler arrives at the sink node of the link (with random arrival time), he/she makes another choice out of a new set of paths from that node to the destination, whose attributes are updated based on the actual arrival time. He/She then again follows the first link of the chosen path, which is not necessarily the second link on the chosen path from the previous decision node. An adaptive path appears to be superior to a non-adaptive path which ignores information on actual arrival time at intermediate nodes, yet the choice is still short-sighted. At each decision node, the next link is chosen based on a path, and thus the fact that he/she can be adaptive at subsequent decision points is not taken into account.

The routing policy model, on the other hand, fully considers future adaptive choices. Generally speaking, a routing policy is a mapping from network states to choices of next link, where the set of network states depends on the assumptions on stochastic networks and information access. In this paper, a routing policy is a mapping $(v, t) \rightarrow \ell$ from node $v$ at arrival time $t$ to next link $\ell \in \mathrm{O}(v)$ where $\mathrm{O}(v)$ is the set of outgoing links of node $v$. For example, denote $e(v, t)$ as the minimum expected travel time of a routing policy from node $v$ at time $t$ to a given destination. A traveler who minimizes expected travel time would choose a link $\ell=(v, w)$ such that $\mathrm{E}\left(\widetilde{\mathrm{T}}_{\ell, \mathrm{t}}+e\left(w, \mathrm{t}+\widetilde{\mathrm{T}}_{\ell, \mathrm{t}}\right)\right)$ is the minimum among all the outgoing links, where $E(\widetilde{X})$ stands for the expected value of random variable $\widetilde{X}$. The second addend $e\left(w, t+\widetilde{T}_{\ell, t}\right)$ is the expected travel time of a routing policy from the sink node $w$ to the destination, and thus future adaptive choices are taken into account. For each support point of the random network, a routing policy will manifest as a path, but the manifested path changes over support points. In this sense, a routing policy can be viewed as a collection of paths, each with a certain probability. The readers are referred to Gao and Chabini (2006) and Gao (2005) for a detailed account of optimal routing policy problems in stochastic time-dependent networks. 


\section{Illustrative Example}

We use an example to clarify the concepts related to the two adaptive choices. Figure 1 gives the topology of the stochastic and time-dependent network and the PMFs of relevant link travel times, where $T_{i}$ denotes the travel time on link $i$. Travelers are going from nodes A to D at departure time 0 . The possible (node, time) pairs a traveler could encounter during the trip are:

$$
(A, 0),\left(B, x_{1}\right),\left(B, y_{1}\right),\left(C, x_{2}\right),\left(C, y_{2}\right)
$$

and the sets of outgoing links for all decision nodes are:

$$
\mathrm{O}(\mathrm{A})=\{0,1\}, \mathrm{O}(\mathrm{B})=\{2,3\}, \mathrm{O}(\mathrm{C})=\{4,5\}
$$

Theoretically the number of routing policies are $2^{5}$, since there are 5 possible (node, time) pairs and each pair can be mapped to two possible next links. However, once a traveler is at node B, the mapping at node $C$ does not affect his/her remaining trip and therefore do not need to be specified. The same argument can be made at node $C$ where the mapping at node $B$ is not needed. Therefore there are 8 routing policies as shown in Figure 1. Note that a path is a special routing policy, such that the mapping from a (node, time) pair is the same regardless of the arrival time. Discussions of calculating attributes of the routing policies can be found in Section 5 ,

We use general symbols for the PMFs, but for illustrative purpose, we make the example simple by assuming $a=f, P_{1}=P_{2}=0.5, x_{0}=x_{1}=$ $x, y_{0}=y_{1}=y$.

Travel times on links 0 and 1 at departure time 0 are random. It is assumed that these two random variables are independent of each other. There are no restrictions on the values of $x$ and $y$, but for illustrative purpose, we assume $x<y$ and denote the situation where link 0 or 1 has a travel time of $x$ as the normal case, and that where link 0 or 1 has a travel time of $y$ as the incident case. Travel times on links 2 and 4 are deterministic, but are dependent on the arrival times at source nodes of the links, which could be either $x$ or $y$. A later arrival time at node $B$ (alternatively C) leads to a longer travel time on link 2 (alternatively 4 ) 


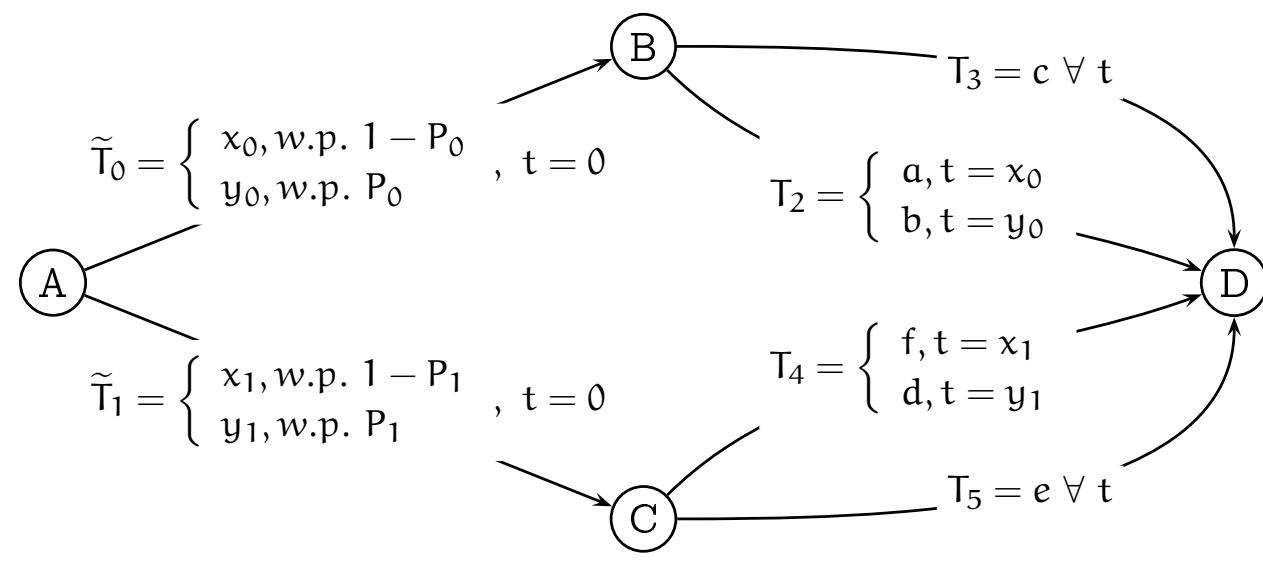

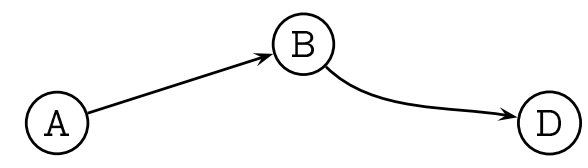

(C)

Policy 1 (Path 1)

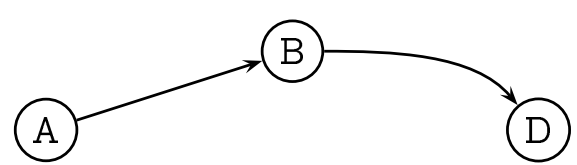

(C)

Policy 4 (Path 2)

(B)

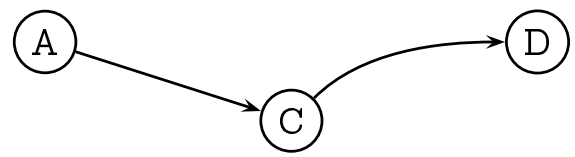

Policy 5 (Path 3)

(B)

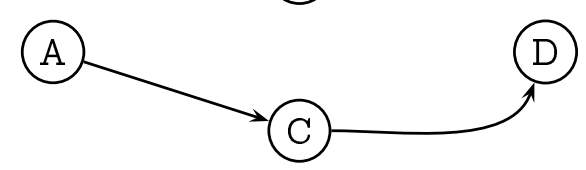

Policy 8 (Path 4)

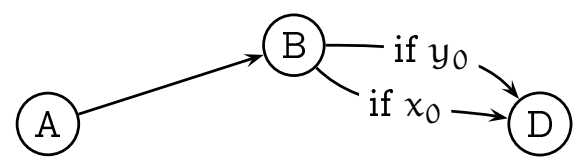

(C)

Policy 2

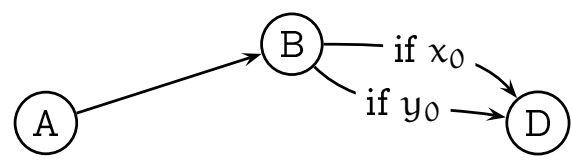

(C)

Policy 3

(B)

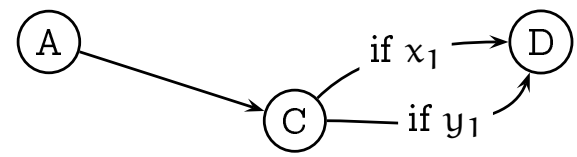

Policy 6

(B)

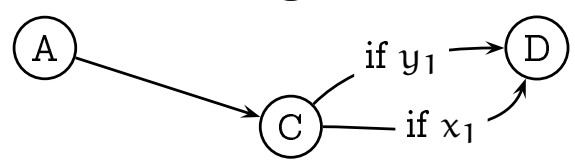

Policy 7

Figure 1: Network 
( $b>a, d>f=a$ ). This could be due to the fact travelers who arrives late (y) are caught in peak traffic, while those with an earlier arrival $(x)$ could have avoided it. Travel times on links 3 and 5 are both deterministic and time-independent.

The relationships among link travel time variables are: $a=f<(a+$ b) $/ 2<\mathrm{e}<\mathrm{c}<\mathrm{b}<\mathrm{d}$. The peak traffic condition on link 4 is more severe than that on link 2, such that the travel time on link 4 at time $y(d)$ is higher than that of link 2 (a). However, both links have diversions. Link 2 has link 3 as the diversion link with a travel time of c, and link 4 has link 5 as the diversion link with a travel time of $e$. Link 5 is a better diversion than link 3 , since $e<\mathrm{c}$.

A traveler has a priori knowledge on the time-dependent link travel time PMFs of all links in the network before a trip starts. During the trip, the traveler obtains additional online information on the actual arrival time at the second node ( $x$ or $y$ ). Depending on the arrival time, the traveler chooses the next link to take to minimize expected travel time.

Consider first the route choice process in an adaptive path model. At node $A$, four paths are available: path 1 with an expected travel time $(x+a+y+b) / 2$, path 2 with an expected travel time $(x+y) / 2+c$, path 3 with an expected travel time $(x+f+y+d) / 2$, and path 4 with an expected travel time $(x+y) / 2+e$. Path 1 has the minimum expected travel time, and thus the traveler takes link 0 which is the first link along that path. The traveler then arrives at node $B$ at either time $x$ or $y$, each with probability 0.5 . If the arrival time is $x$ (off peak), the traveler takes link 2 with a travel time of $a$; and if the arrival time is $y$ (peak), the traveler takes a detour which is link 3 with a travel time of $c$. Therefore the expected travel time from node $A$ to node $D$ by making successive path choices is $(x+a+y+c) / 2$.

Consider next the choice process in a routing policy model. At node $A$, the traveler is actually comparing the attractiveness of links 0 and 1 . The traveler knows that once arriving at the next node, he/she would make a choice based on realized arrive time, therefore it is better to consider all the possible diversions. The optimal routing policy from node B is to take the faster of links 2 and 3: if arrival time is $x$, take link 2 with a travel 
time a; if arrival time is $y$, take link 3 with a travel time c. Similarly, the optimal routing policy at node $C$ is to take the faster of links 4 and 5: if arrival time is $x$, take link 4 with a travel time $f$; if arrival time is $y$, take link 5 with a travel time $e$. With this calculation in hand, the traveler evaluates at node $A$ and decides that taking link 1 is optimal, since $(x+a+y+e) / 2<(x+f+y+c) / 2$ (note that $a=f)$. Recalling that the expected travel time of making successive path choices is $(x+a+y+c) / 2$, the optimal routing policy is thus more efficient as a result of considering future adaptive possibilities.

\section{Model Specifications}

In this section we present discrete choice model formulations for the previously discussed adaptive path and routing policy choices. Note that in the data for model estimation, only the manifested path is observed. Each path observation $i$ of individual $n$ is an ordered set of chosen links $\mathcal{I}_{i}$. Also known are the departure time and the arrival time $t$ at the source node $v$ of each link $\ell \in \mathcal{I}_{i}$. Such information are is available, for example, from Global Positioning System (GPS), see Bierlaire and Frejinger (2007) for a discussion on route choice data.

\subsection{Adaptive Path Choice Model}

This model assumes that a traveler chooses at the source node $v$ of each observed link $\ell \in \mathcal{I}_{i}$ a path $p$ from $v$ to the destination. We therefore define an individual and time specific choice $\operatorname{set} \mathcal{C}_{v t n}$ of paths from $v$ to the destination. Hence, for each observation there are as many choice sets as there are links in the observed path.

The probability of an observation is defined as the product of the probability of choosing each link $\ell$ in the observed path, conditional on arrival time $t$ at the source node:

$$
P_{n}(i)=\prod_{\ell \in \mathcal{I}_{i}} P_{n}(\ell \mid t, v)=\prod_{\ell \in \mathcal{I}_{i}} \sum_{p \in \mathcal{C}_{v t n}} P(\ell \mid p) P\left(p \mid \mathcal{C}_{v t n} ; \beta\right)
$$


$P_{n}(\ell \mid t, v)$ is defined by the sum of the probabilities for each path that begins with $\ell$. The path choice model $P\left(p \mid \mathcal{C}_{v t n} ; \beta\right)$ ( $\beta$ denotes the vector of parameters to be estimated) is therefore multiplied with a binary variable $P(\ell \mid p)$ that equals one if the first link in path $p$ is $\ell$ and zero otherwise. Note that the path choice can be modeled with any of the existing non-adaptive models.

\subsection{Routing Policy Choice Model}

Consider the model for the choice of routing policy among a choice set $\mathcal{G}$ of routing policies at the origin. Note that the adaptive behavior is already captured in the definition of a routing policy. The choice of routing policy is latent and only the manifested path is observable. A support point is fully defined by the realized travel times on all random links. We assume that the realized support point for each observation is known to the modeler through, for example, adequately dispersed GPS observations or probe vehicles that cover all random links. The traveler does not know the realized support point at the origin; his/her informationn access is defined in the routing policy, and in this paper it is the arrival times at decision nodes. We model the the probability of a path observation conditional on support point $r$ and choice set of routing policies $\mathcal{G}$ as

$$
\mathrm{P}_{\mathrm{n}}(\mathfrak{i} \mid \mathrm{r})=\sum_{\gamma \in \mathcal{G}} \mathrm{P}(\mathrm{i} \mid \gamma, \mathrm{r}) \mathrm{P}(\gamma \mid \mathcal{G})
$$

where $\gamma$ is a routing policy. As described in Section 3, for a given support point a routing policy is manifested as a path. However, several different routing policies can be manifested as the same path. We therefore sum over all routing policies in $\mathcal{G}$ and multiply the routing policy choice model $\mathrm{P}(\gamma \mid \mathcal{G})$ with a binary variable $\mathrm{P}(i \mid \gamma, r)$ that equals one if $i$ corresponds to $\gamma$ for support point $r$ and zero otherwise.

Gao (2005) propose the policy size logit to model $\mathrm{P}(\gamma \mid \mathcal{G})$ which is the routing policy version of the path size logit model (Ben-Akiva and Ramming, 1998; Ben-Akiva and Bierlaire, 1999). It adds a term, policy size (PoS), to the deterministic utilities that corrects for correlation among 
routing policies. The model is defined as

$$
\mathrm{P}(\gamma)=\frac{e^{\ln \operatorname{PoS}_{\gamma}+V_{\gamma}}}{\sum_{k \in \mathcal{G}} e^{\ln \operatorname{PoS}_{k}+V_{k}}}
$$

where $V_{\gamma}$ is the deterministic utility of $\gamma$ and the formulation of PoS

$$
\operatorname{PoS}_{\gamma}=\sum_{r=1}^{R}\left(\sum_{\ell \in \mathcal{I}_{\gamma}^{r}}\left(\frac{\mathrm{T}_{\ell}^{r}}{T_{\gamma}^{r}}\right) \frac{1}{M_{\ell}^{r}}\right) \mathrm{P}(\mathrm{r})
$$

may be viewed as "expected path size" with notations

$\mathrm{R}$ : number of support points of link travel time distribution;

$\mathcal{I}_{\gamma}^{r}$ : set of links of the realized path of routing policy $\gamma$ for support point $r$;

$\mathrm{T}_{\ell}^{r}$ : travel time of link $\ell$ for support point $r$;

$\mathrm{T}_{\gamma}^{r}$ : realized travel time of routing policy $\gamma$ for support point $r$;

$M_{\ell}^{r}$ : number of routing policies using link $\ell$ for support point $r$;

$\mathrm{P}(\mathrm{r})$ : probability of support point $r$.

Note that if the support point is unknown due to data unavailability, a path cannot unambiguously be matched with a given routing policy. The model presented in Equation (2) can then be generalized to

$$
\mathrm{P}_{\mathrm{n}}(\mathfrak{i})=\sum_{\gamma \in \mathcal{G}} \mathrm{P}(\mathfrak{i} \mid \gamma) \mathrm{P}(\gamma \mid \mathcal{G})=\sum_{\gamma \in \mathcal{G}} \sum_{r=1}^{\mathrm{R}} \mathrm{P}(\mathfrak{i} \mid \gamma, r) \mathrm{P}(\gamma \mid \mathcal{G})
$$

\section{$5 \quad$ Numerical Results}

We carry out numerical tests of the proposed adaptive route choice models on a hypothetical network. The objectives of the tests are to: 1) demonstrate the feasibility of estimating the two adaptive route choice models; and 2) gain insights into the adaptive route choice models by comparing prediction results.

\subsection{Test Settings}

The test network is the same as discussed in Section 3, As explained earlier, there are eight routing policies. The travel time of each routing policy is 
a discrete random variable with two possible values. For example, the travel time of the routing policy 6 (the one discussed in Section (3) can be either $x_{1}+f$ or $y_{1}+d$, with probability $1-P_{1}$ and $P_{1}$ respectively. The expected travel time and standard deviation of the routing policy then can be calculated.

The calculation of policy size is more involved. As shown in Equation (44), policy size is the expected value of path sizes over all support points of the random network. As there are two random links in the network each with two possible realizations of travel times, there are altogether four support points. Let $\left(T_{0}, T_{1}\right)$ represents a support point where $T_{0}$ and $T_{1}$ are realized travel times on links 0 and 1 respectively. The four support points are then $\left(x_{0}, x_{1}\right),\left(x_{0}, y_{1}\right),\left(y_{0}, x_{1}\right),\left(y_{0}, y_{1}\right)$. In the following, we will use support point $\left(x_{0}, x_{1}\right)$ as an example to illustrate how policy size is calculated.

Consider first the mapping from routing policies to paths. For example, in support point $\left(x_{0}, x_{1}\right)$, routing policy 2 takes link 2 at node $B$ at arrival time $x_{0}$, and therefore is manifested as path 1 . Let $\gamma$ be a routing policy and $p$ a path, we obtain manifestation of all routing policies as follows:

$$
\gamma_{1} \rightarrow p_{1}, \gamma_{2} \rightarrow p_{1}, \gamma_{3} \rightarrow p_{2}, \gamma_{4} \rightarrow p_{2}, \gamma_{5} \rightarrow p_{3}, \gamma_{6} \rightarrow p_{3}, \gamma_{7} \rightarrow p_{4}, \gamma_{8} \rightarrow p_{4}
$$

Once the manifested path is known, we can count the number of routing policies that use a given link in support point $\left(x_{0}, x_{1}\right)$ as follows.

$$
\begin{aligned}
& M_{\ell_{0}}=4\left(\gamma_{1}, \gamma_{2}, \gamma_{3}, \gamma_{4}\right) \\
& M_{\ell_{1}}=4\left(\gamma_{5}, \gamma_{6}, \gamma_{7}, \gamma_{8}\right) \\
& M_{\ell_{2}}=2\left(\gamma_{1}, \gamma_{2}\right) \\
& M_{\ell_{3}}=2\left(\gamma_{3}, \gamma_{4}\right) \\
& M_{\ell_{4}}=2\left(\gamma_{5}, \gamma_{6}\right) \\
& M_{\ell_{5}}=2\left(\gamma_{7}, \gamma_{8}\right)
\end{aligned}
$$


The routing policy sizes in support point $\left(x_{0}, x_{1}\right)$ are then

$$
\begin{aligned}
& \mathrm{PoS}_{1}=\frac{x_{0}}{x_{0}+a} \frac{1}{4}+\frac{a}{x_{0}+a} \frac{1}{2} \\
& \mathrm{PoS}_{2}=\frac{x_{0}}{x_{0}+a} \frac{1}{4}+\frac{a}{x_{0}+a} \frac{1}{2} \\
& \mathrm{PoS}_{3}=\frac{x_{0}}{x_{0}+c} \frac{1}{4}+\frac{c}{x_{0}+c} \frac{1}{2} \\
& \mathrm{PoS}_{4}=\frac{x_{0}}{x_{0}+c} \frac{1}{4}+\frac{c}{x_{0}+c} \frac{1}{2} \\
& \mathrm{PoS}_{5}=\frac{x_{1}}{x_{1}+f} \frac{1}{4}+\frac{f}{x_{1}+f} \frac{1}{2} \\
& \mathrm{PoS}_{6}=\frac{x_{1}}{x_{1}+f} \frac{1}{4}+\frac{f}{x_{1}+f} \frac{1}{2} \\
& \mathrm{PoS}_{7}=\frac{x_{1}}{x_{1}+e} \frac{1}{4}+\frac{e}{x_{1}+e} \frac{1}{2} \\
& \operatorname{PoS}_{8}=\frac{x_{1}}{x_{1}+e} \frac{1}{4}+\frac{e}{x_{1}+e} \frac{1}{2}
\end{aligned}
$$

Similarly we can calculate the policy sizes in all other support points. Take the expectation over the four support points and we obtain the final policy sizes.

We also need to calculate path sizes at the origin node $A$ to be used in both the non-adaptive path model and the adaptive path model. Path sizes are based on expected travel times.

$$
\begin{aligned}
P_{1} & =\frac{x_{0}\left(1-P_{0}\right)+y_{0} P_{0}}{\left(x_{0}+a\right)\left(1-P_{0}\right)+\left(y_{0}+b\right) P_{0}} \frac{1}{2}+\frac{a\left(1-P_{0}\right)+b P_{0}}{\left(x_{0}+a\right)\left(1-P_{0}\right)+\left(y_{0}+b\right) P_{0}} \\
P_{2} & =\frac{x_{0}\left(1-P_{0}\right)+y_{0} P_{0}}{x_{0}\left(1-P_{0}\right)+y_{0} P_{0}+c} \frac{1}{2}+\frac{c}{x_{0}\left(1-P_{0}\right)+y_{0} P_{0}+c} \\
P S_{3} & =\frac{x_{1}\left(1-P_{1}\right)+y_{1} P_{1}}{\left(x_{1}+f\right)\left(1-P_{1}\right)+\left(y_{1}+d\right) P_{0}} \frac{1}{2}+\frac{f\left(1-P_{1}\right)+d P_{1}}{\left(x_{1}+f\right)\left(1-P_{1}\right)+\left(y_{1}+d\right) P_{1}} \\
P_{4} & =\frac{x_{1}\left(1-P_{1}\right)+y_{1} P_{1}}{x_{1}\left(1-P_{1}\right)+y_{1} P_{1}+e} \frac{1}{2}+\frac{e}{x_{1}\left(1-P_{1}\right)+y_{1} P_{1}+e}
\end{aligned}
$$

\subsection{Observation Generation}

We assume the routing policy choice model of Equation (3) is the true model. We move the policy size into the deterministic utility function and 
specify it with the following postulated coefficients

$$
\mathrm{V}_{\gamma}=1.0 \ln \mathrm{PoS}_{\gamma}-0.4 \text { ExpectedTime }_{\gamma}-0.1 \mathrm{TimeSTD}_{\gamma}, \forall \gamma \in \mathcal{G}
$$

A routing policy is not observable, and only the manifested path for a given support point is observed. We specify the range of link travel time to be $[10,40](\mathrm{min})$. To generate one path observation from the policy choice model, we follow the steps:

1. Sample a number from a uniform distribution between 10 and 40 for each link travel time variable: $a, b, c, d, e, f$;

2. Sample a number from a uniform distribution between 0 and 1 for each link travel time probability variable: $x_{0}, y_{0}, x_{1}, y_{1}$;

3. Calculate $P\left(\gamma_{k}\right)$, the choice probability of routing policy $k, \forall k$ using the policy-size logit model (Equation (3) $)$;

4. Calculate $Q_{k}$, the cumulative probability of choosing policies $\gamma_{1}, \gamma_{2}, \ldots, \gamma_{k}$, $\mathrm{k}=0,1, \ldots, 8$, where $\mathrm{Q}_{0}=0$ and $\mathrm{Q}_{8}=1$;

5. Sample a number $r$ from a uniform distribution between 0 and 1 , and $\gamma_{k}$ is chosen if $Q_{k-1}<r<Q_{k}$;

6. Calculate $S_{k}$, the cumulative probability of support points $r_{1}, r_{2}, \ldots, r_{k}$, $k=0,1, \ldots, 4$, where $S_{0}=0$ and $S_{4}=1$;

7. Sample a number $r^{\prime}$ from a uniform distribution between 0 and 1 ; and support point $r_{k}$ is realized if $S_{k-1}<r^{\prime}<S_{k}$;

8. The chosen routing policy is manifested as a path depending on the support point, using a similar logic as in the mapping of Equation (6)

\subsection{Estimation}

Three models are estimated based on the generated path observations:

1. Routing policy choice model (Equation (2)) with policy size logit; 
2. Adaptive path choice model (Equation (1I)) with path size logit;

3. Non-adaptive path choice model with path size logit.

The deterministic utility functions have a linear-in-parameters specification of the same attributes as the true model, namely, expected travel time, travel time standard deviation and path (policy) size.

In the adaptive path choice model, the choice probability of a path is the product of choosing all links along the path. The log likelihood of the path is then the sum of the log likelihood of all the links. Therefore we can treat each chosen link as an observation, and the link choice probability is the sum of choice probabilities of paths beginning with the the link. Note that the path attributes are dependent on the realized arrival time at the upstream node of the link. For example, assume $\gamma_{2}$ is chosen and a travel time of $x_{0}$ is realized on link 0 , and thus the manifested path is path 1 . Path 1 is composed of two links: 0 and 2. Choice probability of link 0 is the sum of those of paths 1 and 2. At time 0 and node $A$, travel time on link 0 is still random and therefore the expected travel time of path 1 is $\left(x_{0}+a\right)\left(1-P_{0}\right)+\left(y_{0}+b\right) P_{0}$ and that of path 2 is $\left(x_{0}+a\right)\left(1-P_{0}\right)+\left(y_{0}+c\right) P_{0}$. Now consider the choice of link 2. At time $x_{0}$ and node $B$, travel time on link 2 (coinciding with one of the path alternatives out of node B) is fixed as $a$, and such value should be used in the observation data.

All models are estimated with BIOGEME (Bierlaire, 2003; Bierlaire, 2005). The estimation results are shown in Table 1, The coefficient estimates of routing policy choice model are not significantly different from the postulated values which shows that a policy choice model can be estimated based on path observations using Equation (21). The coefficient estimates of the other two models have their appropriate signs and are significantly different from zero. As expected the model fit of the routing policy model is better than the adaptive path model and the non-adaptive path model has the worst model fit.

\subsection{Prediction}

The three estimated models are applied to predict route choices in the same topological network, but with a fixed set of hypothetical link travel time 


\begin{tabular}{|l|c|c|c|}
\hline & Policy & $\begin{array}{c}\text { Adaptive } \\
\text { path }\end{array}$ & $\begin{array}{c}\text { Non-adaptive } \\
\text { path }\end{array}$ \\
\hline$\widehat{\beta}_{\text {PS }}$ & 1.03 & 1.23 & 2.75 \\
Std error & 0.452 & 0.437 & 0.344 \\
T-test & 2.28 & 2.80 & 8.00 \\
\hline$\widehat{\beta}_{\text {exptime }}$ & -0.402 & -0.28 & -0.265 \\
Std error & 0.00805 & 0.00467 & 0.0049 \\
T-test & -49.97 & -60.00 & -54.02 \\
\hline$\widehat{\beta}_{\text {stdtime }}$ & -0.108 & -0.071 & -0.0451 \\
Std error & 0.00857 & 0.00923 & 0.00643 \\
T-test & -12.60 & -7.69 & -7.02 \\
\hline \hline Final log-likelihood & -3257.097 & -3536.324 & -3932.998 \\
Adj. rho-square & 0.608 & 0.574 & 0.527 \\
\hline Number of observed paths: 6000 & \\
Null log-likelihood: -8317.766 & \\
BIOGEME (Bierlaire, 2003; Bierlaire, 2005) has been \\
used for all model estimations \\
\hline
\end{tabular}

Table 1: Estimation Results 
variables as follows:

$$
\begin{aligned}
& a=f=10, b=30, c=26, d=38, e=22 \\
& x_{0}=x_{1}=14, y_{0}=y_{1}=18 \\
& P_{0}=P_{1}=P
\end{aligned}
$$

The value of $P$ is a parameter of the prediction tests and varies from 0 to 1 , with an increment of 0.1 . The values of the link travel time variables satisfy the condition in Section 3 , i.e. $a=f<(a+b) / 2<e<c<d$. Therefore the analysis in Section 3 applies here: path 1 is the minimum expected travel time path; links 2 and 4 have the same travel time under normal condition (when $x_{0}$ or $x_{1}$ is realized); link 4 is more congested than link 2 under incident condition (when $y_{0}$ or $y_{1}$ is realized); links 3 and 5 are diversions for links 2 and 4 in incident condition, and link 5 is a better diversion than link $3(e<c)$.

Since the network is stochastic with all the support points known, we obtain distributions of variables such as path shares, path travel times, origin-destination travel time and so forth. We take expectations of these variabls over the four support points, where the probability of each support is a function of $P$. We present the summary statistics (mean and/or standard deviation) to gain a high-level understanding of the results.

Figure 2 shows the expected shares of all four paths. Each subgraph is for a path, and results from all three models are plotted as functions of incident probability $P$. Recall that paths 1 and 3 contain the links that can be affected by the incidents due to the time-dependency of their travel times, while paths 2 and 4 contain the respective diversion links that are not affected by the incidents. Therefore it is intuitively correct that for all three models, the shares of paths 1 and 3 are decreasing functions of $P$, while shares of paths 2 and 4 are increasing functions of $P$.

In order to better appreciate the differences among the three models, we aggregate the results from Figure 2 to yield data for Figure 3, where the expected shares of going left and right at the origin node are plotted. Recall that the right branch has a better diversion (link 5). In the routing policy model, as $\mathrm{P}$ increases, the importance of diversion becomes more significant and therefore more flow goes to the right. In the two path models, as $\mathrm{P}$ 
increases, the left share first increases and then decreases. This is because when $\mathrm{P}=0$, both paths 1 and 3 (belonging to the left and right branches respectively) have the same minimum travel time $(a=f)$ and zero standard deviation. While as $P$ increases, path 3 has higher disutility $(d>b)$, and therefore path 1 is more dominant and gains more share. However, as $P$ increases to a certain value, path 4 becomes the path with minimum disutility, and thus the right share starts to increase.

If we inspect Figures 2 and 3 together, we find that although the leftright shares of non-adaptive and adaptive path models are roughly the same, the distribution of the flows at the second nodes are different. This is because the adaptive path model redistributes flows at the second nodes depending on the actual arrival times. On the other hand, both the adaptive path model and non-adaptive path model predict more flow taking the left branch than the routing policy model does. This is because future diversion possibility is not considered in either of the models, and the branch with less expected path travel time (path 1) is favored, although link 3 is a worse diversion. In another word, the routing policy model better capture the option value of diversion than the adaptive path model.

Figure 4 shows the expected value and standard deviation of average path travel time where the average is taken over all four paths weighted by path shares. As $P$ is approaching 0 or 1 , links 0 and 1 are more likely to be in the same condition and the network is less stochastic than when $\mathrm{P}$ is in the middle. The figure shows that as $\mathrm{P}$ approaches the middle point between 0 and 1 , the two adaptive models and non-adaptive models are farther away from each other in terms of expected average travel time. This is in accordance with the intuition that being adaptive is more advantageous when the network is more uncertain.

Figure 4 also shows that adaptive path and routing policy models have similar expected average travel time, but their standard deviations are quite different. This is because the effect of a diversion is two-fold. A better diversion provides shorter travel time under incident condition. On the other hand, under normal condition it also results in more flow moving from the faster link, because its disutility is not as far away from the faster link as the worse diversion. This results in longer travel time averaged 
over the faster link and the diversion. Since the adaptive path model predicts more flow to the left branch (worse diversion) than the routing policy model, it has longer travel time under incident condition, but shorter travel time under normal condition. Hence both models predict roughly the same expected average travel time, but the adaptive path model has larger standard deviations.

\section{Conclusions and Future Directions}

This paper develops the first econometric estimator for the routing policy choice model and demonstrates the feasibility of estimating the model. A routing policy in general is a decision rule that maps from all possible network states to next links out of decision nodes. It collapses to a path in a deterministic networks. The concept of routing policy explicitly captures travelers' route choice adjustments according to information on realized network conditions in stochastic time-dependent networks. Since the information component is embedded in the defintion of a routing policy, the estimator of the routing policy model is a general one and can be applied to a large variety of information situations. This paper demonstrates one of the information situations: the realized arrival times at decision nodes. Other information situations will be the subjects of future research.

The routing policy choice model is also compared to the adaptive path model, which is a natural approach to an adaptive route choice model. An adaptive path model is actually a sequence of path choice models applied at intermediate decision nodes; while a routing policy choice model is one model at the origin where the alternatives are routing policies. Prediction results show that the routing policy model captures better the option value of diversion than the adaptive path model because of the foresight of a routing policy. When the network is more stochastic, the difference between the two adaptive models and the non-adaptive model is larger in terms of expected travel time. As expected, this result indicates that the benefit of being adaptive is more significant in a more uncertain network.

Future research directions would include estimation of the routing policy choice model with field data, study of other information situations and 
investigation of alternative methods to capture the overlapping of routing policies.

\section{References}

Abdel-Aty, M. A. and Abdalla, M. F. (2006). Examination of multiple mode/route-choice paradigms under atis, IEEE Transactions on Intelligent Transportation Systems 7(3): 332-348.

Abdel-Aty, M. and Abdalla, M. F. (2004). Modeling drivers' diversions from normal routes under atis using generalized estimating equations and binomial probit link function, Transportation 31: 327-348.

Balakrishna, R. (2006). Off-line calibration of dynamic traffic assignment models, $\mathrm{PhD}$ thesis, Massachusetts Institute of Technology, Cambridge, MA, USA.

Balakrishna, R., Ben-Akiva, M. and Koutsopoulos, H. N. (2007). Offline calibration of dynamic traffic assignment: simultaneous demandsupply estimation, Transportation Research Record 2003.

Bekhor, S., Ben-Akiva, M. and Ramming, M. (2002). Adaptation of logit kernel to route choice situation, Transportation Research Record 1805: $78-85$.

Ben-Akiva, M. and Bierlaire, M. (1999). Discrete choice methods and their applications to short-term travel decisions, in R. Hall (ed.), Handbook of Transportation Science, Kluwer, pp. 5-34.

Ben-Akiva, M., Bierlaire, M., Koutsopoulos, H. N. and Mishalani, R. (2002). Real-time simulation of traffic demand-supply interactions within dynamit, in M. Gendreau and P. Marcotte (eds), Transportation and Network Analysis: Current Trends. Miscellenea in Honor of Michael Florian, Kluwer, pp. 19-36. 
Ben-Akiva, M. and Ramming, S. (1998). Lecture notes: Discrete choice models of traveler behavior in networks. Prepared for Advanced Methods for Planning and Management of Transportation Networks. Capri, Italy.

Bierlaire, M. (2003). Biogeme: a free package for the estimation of discrete choice models, Proceedings of the 3rd Swiss Transport Research Conference, Ascona, Switzerland.

Bierlaire, M. (2005). An introduction to Biogeme version 1.4. http://biogeme.epfl.ch.

Bierlaire, M. and Frejinger, E. (2007). Route choice modeling with networkfree data, Transportation Research Part C . Accepted for publication.

Bogers, E. A. I., Viti, F. and Hoogendoorn, S. P. (2005). Joint modeling of advanced travel information service, habit, and learning impacts on route choice by laboratory simulator experiments, Transportation Research Record 1926: 189-197.

Cascetta, E., Nuzzolo, A., Russo, F. and Vitetta, A. (1996). A modified logit route choice model overcoming path overlapping problems. Specification and some calibration results for interurban networks, in J. B. Lesort (ed.), Proceedings of the 13th International Symposium on Transportation and Traffic Theory, Lyon, France.

Frejinger, E. and Bierlaire, M. (2007). Capturing correlation with subnetworks in route choice models, Transportation Research Part $B$ 41(3): 363-378.

Gao, S. (2005). Optimal Adaptive Routing and Traffic Assignment in Stochasitc Time-Dependent Networks, PhD thesis, Massachusetts Institute of Technology.

Gao, S. and Chabini, I. (2006). Optimal routing policy problems in stochastic time-dependent networks, Transportation Research Part B 40(2): 93-122. 
Hall, R. W. (1986). The fastest path through a network with random time-dependent travel times, Transportation Science 20(3): 91-101.

Mahmassani, H. (2001). Dynamic network traffic assignment and simulation methodology for advanced system management applications, Network and Spatial Economics 1: 267-292.

Mahmassani, H. and Liu, Y. (1999). Dynamics of commuting decision behavior under advanced traveler information systems, Transportation Research Part C 7(2/3): 91-107.

Marcotte, P. and Nguyen, S. (1998). Hyperpath formulations of traffic assignment problems, in P. Marcotte and S. Nguyen (eds), Equilibrium and Advanced Transportation Modeling, Kluwer Academic Publishers, pp. 175-199.

Miller-Hooks, E. (2001). Adaptive least-expected time paths in stochastic, time-varying transportation and data networks, Networks 37(1): 3552.

Miller-Hooks, E. D. and Mahmassani, H. S. (2000). Least expected time paths in stochastic, time-varying transportation networks, Transportation Science 34(2): 198-215.

Peeta, S. and Yu, J. W. (2005). A hybrid model for driver route choice incorporating en-route attributes and real-time information effects, Networks and Spatial Enonomics 5: 21-40.

Polychronopoulos, G. and Tsitsiklis, J. N. (1996). Stochastic shortest path problems with recourse, Networks 27: 133-143.

Polydoropoulou, A., Ben-Akiva, M., Khattack, A. and Lauprete, G. (1996). Modling revealed and stated en-route travel response to adavanced traveler information systems, Transportation Research Record 1537: 38-45.

Pretolani, D. (2000). A directed hyperpath model for random time dependent shortest paths, European Journal of Operational Research 123: 315-324. 
Ramming, M. (2001). Network Knowledge and Route Choice, PhD thesis, Massachusetts Institute of Technology, Cambridge, MA, USA.

Srinivasan, K. K. and Mahmassani, H. (2003). Analyzing heterogeneity and unobserved structural effects in route-switching behavior under atis: a dynamic kernal logit formulation, Transportation Research Part $B$ 37: 793-814.

Ukkusuri, S. V. and Patil, G. R. (2006). Exploring user behavior in online network equilibrium problems, 11th International Conference on Travel Behavior Research Compendium of Papers CD-ROM, Kyoto, Japan.

Vovsha, P. and Bekhor, S. (1998). Link-nested logit model of route choice, Overcoming route overlapping problem, Transportation Research Record 1645: 133-142.

Waller, S. T. and Ziliaskopoulos, A. K. (2002). On the online shortest path problem with limited arc cost dependencies, Networks 40(4): 216-227. 

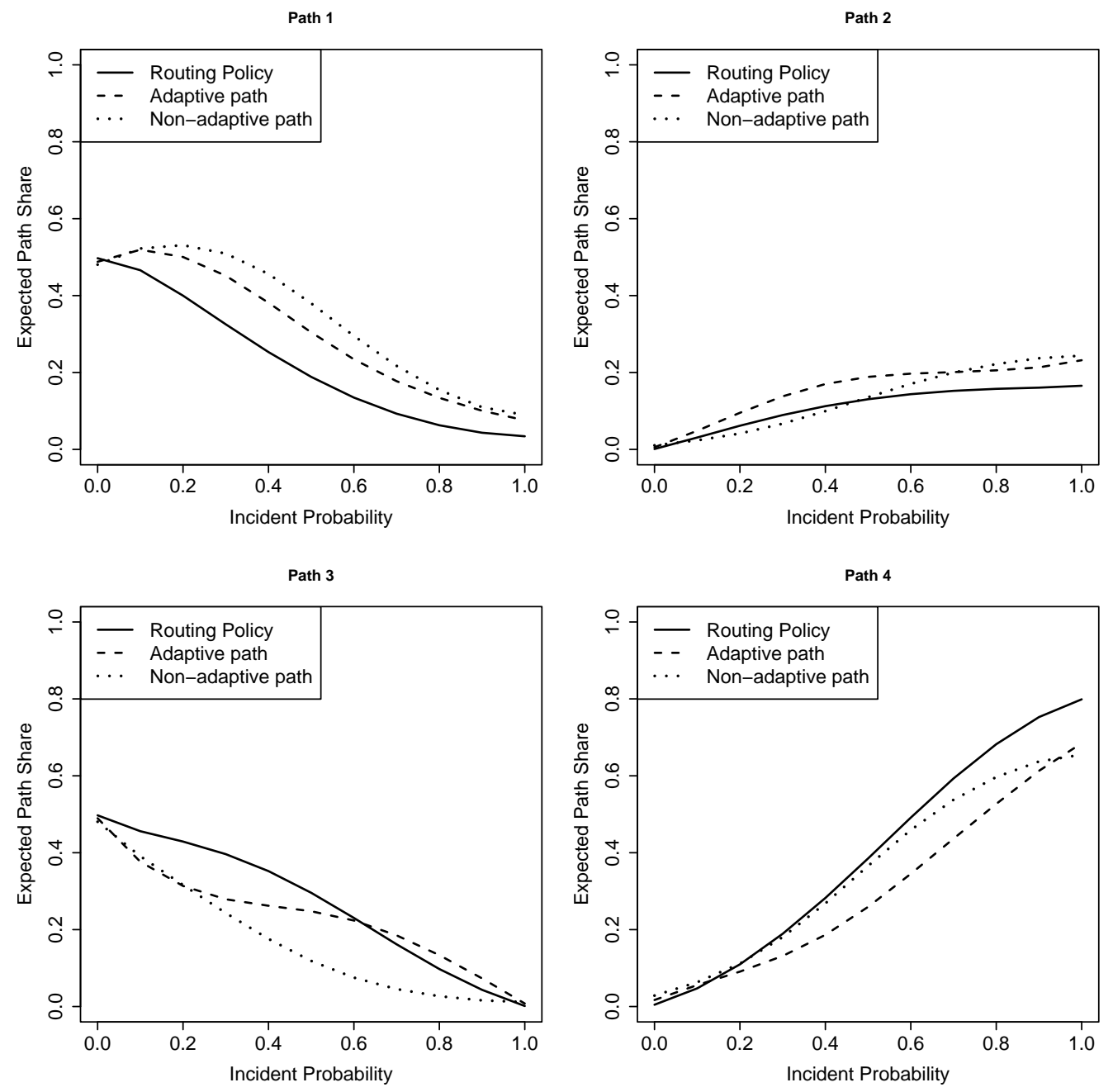

Figure 2: Expected Path Shares 

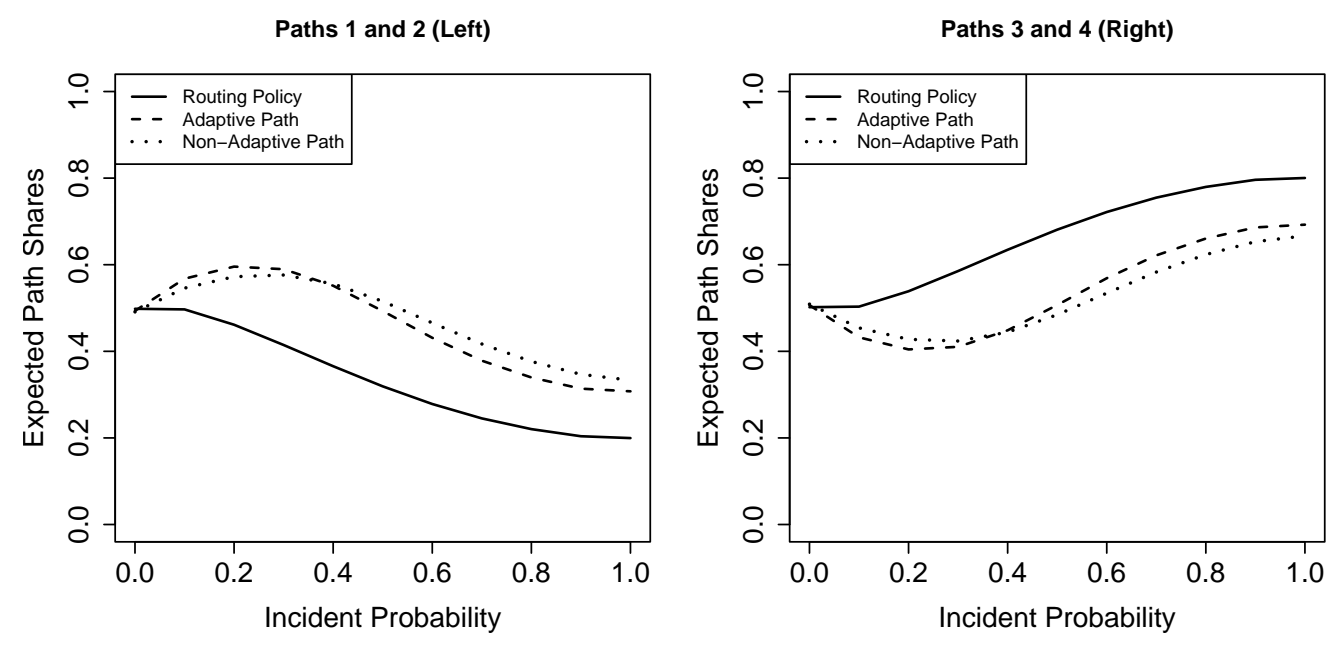

Figure 3: Expected Shares for Left and Right at Origin
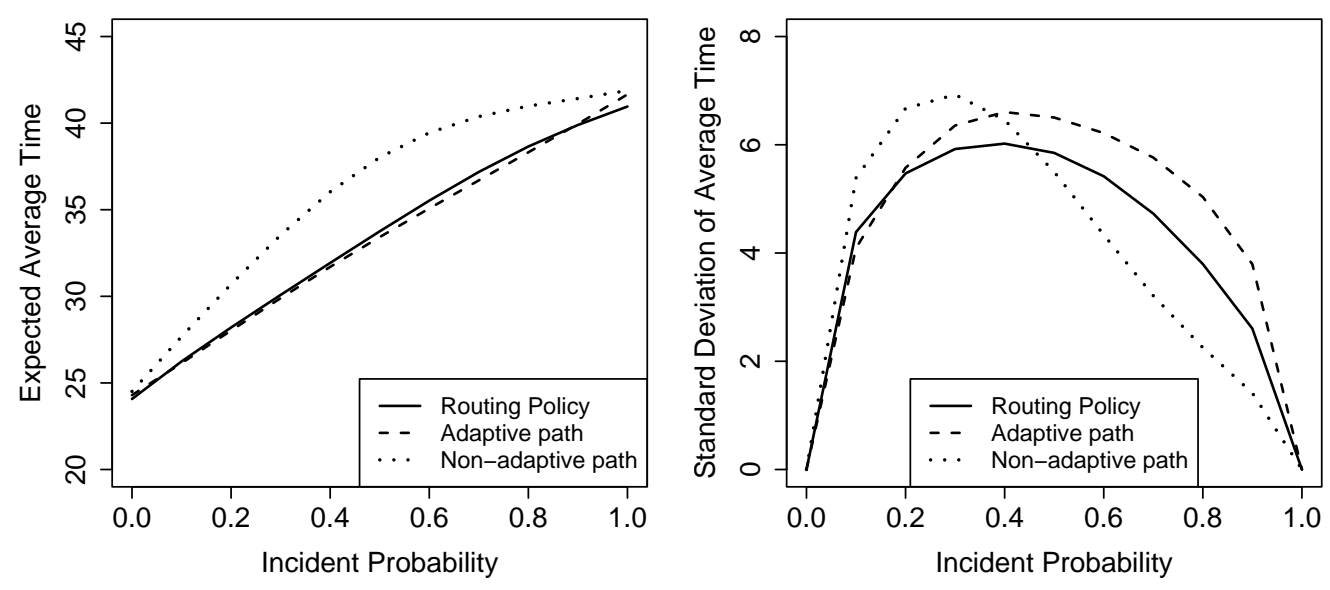

Figure 4: Average Time (Expected Value and Standard Deviation) 